\title{
Trabajo Social e Historia Oral, apuntes para una coincidencia: intervención con adultos mayores
}

\author{
Joel Verdugo-Córdova*
}

Los confines del pasado recuperable mediante la historia oral se reducen inexorablemente día a día por obra de la muerte... (Paul Thompson)

\begin{abstract}
Resumen
En este escrito reflexiono sobre algunos elementos que pudieran ser considerados para la realización de proyectos conjuntos entre el trabajo social y la historia oral. Me refiero a la utilización de la entrevista en profundidad según el método biográfico, herramienta básica de la historia oral como instrumento posibilitador de la rememoración y el redescubrimiento, a partir de la voz de los otros, de las configuraciones y puntos de vista que tienen los protagonistas de su experiencia individual y colectiva. Esta cualidad de la entrevista, en manos del trabajo social, por ejemplo, aplicada en grupos humanos vulnerables (centros geriátricos) pudiera favorecer el incremento en la calidad de vida de los usuarios.
\end{abstract}

\begin{abstract}
With this paper I think some elements could be considered for joint projects between social work and oral history. I mean the use of the in-depth interview as biographical method, a basic tool of oral history as an instrument and enabler of remembering and rediscovery, from the voices of others, settings and views that have the protagonists as individuals and collective experience. This interview's attribute, in the hands of social work, for example, applied in vulnerable human groups (nursing homes) would favor an increase in the quality of life of the users.
\end{abstract}

\section{Introducción}

En el presente artículo reflexionaré sobre las posibles coincidencias entre el trabajo social y la historia oral; además de revisar cómo en la actualidad los préstamos entre las distintas ramas de las ciencias sociales plantean soluciones multidisciplinarias para problemas concretos.
La historia oral como práctica pertinente para otorgarle voz a los "sin voz" y recobrar las riquezas de la vida social a partir de las experiencias individuales y colectivas por medio de la entrevista. También puede constituirse en manos del trabajo social, como un instrumento útil en proyectos concretos que tengan que ver con el aumento de calidad de vida en grupos humanos vulnerables.

En un primer momento reflexionaré sobre los conceptos de trabajo social e historia oral, para después concluir con una breve mención de un proyecto conjunto.

\section{Trabajo Social}

La evolución conceptual y metodológica del trabajo social como disciplina, digamos científica, ha bregado desde la ruptura con prácticas de misericordia y filantropía, hasta el debate epistemológico con corrientes teóricas abrevadas de las ciencias sociales.

Es hasta tiempos recientes, apenas el siglo XX, cuando el trabajo social logra una construcción, más o menos acabada, de su sistematización. El desasosiego que provocan en las sociedades temas como los desvalidos del mundo, la procuración del bienestar de los grupos humanos, los principios de los derechos humanos y la justicia social; también son los principales puntos que desarrolla el trabajo social.

Siguiendo a Flores y Martínez (2006), advertimos que en los campos de acción del trabajo social, los propiamente tradicionales y los nuevos, deben ser motivo de atención e investigación tanto la profesión como la demanda de distintos sectores implicados en la diversidad de problemas sociales y constructores de necesidades humanas, objetivizadas y sentidas y, en

*Departamento de Trabajo Social. Universidad de Sonora. jverdugo@sociales.uson.mx 


\section{就 Scovido}

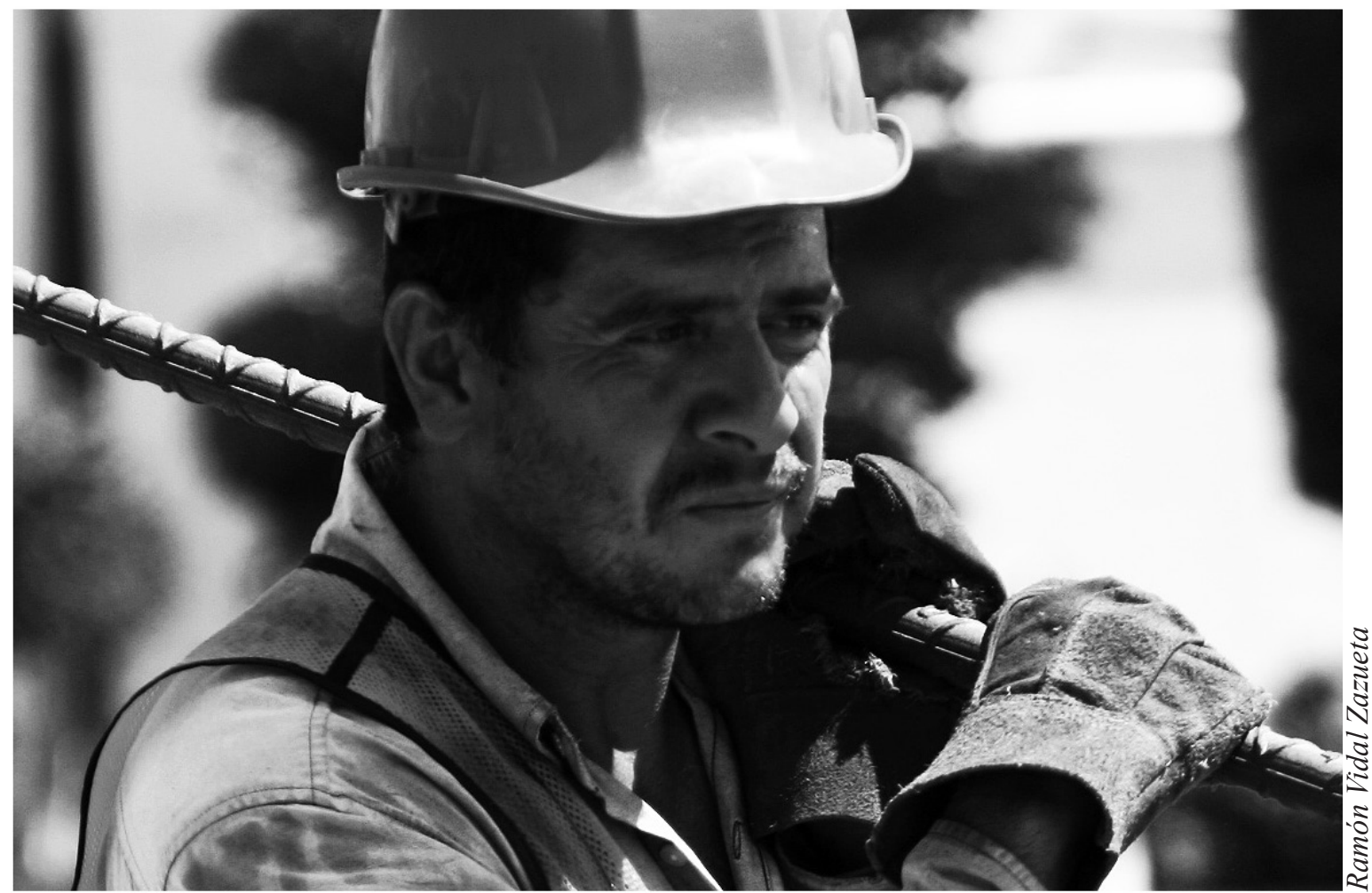

consecuencia, el ejercicio de sus derechos sociales, civiles y humanos. Entre estos sectores se destacan: minorías étnicas, mujeres, niños, jóvenes y ancianos, entre otros.

Por consiguiente resulta cada vez más destacado para el trabajo social, en el marco de las ciencias sociales, sustentar su práctica profesional, en el a veces áspero torbellino de la teoría social, ya que siguiendo a Howel (citado por Del Valle y Ramella, S/F): "cuando a la práctica profesional le quitamos la teoría sólo queda el sentido común y la experiencia acumulada que, si bien pueden ser un bagaje importante, son insuficientes para configurar una disciplina que aspira a los más altos niveles de la formación universitaria.” (pp. 2-3). Es por lo anterior que resulta importante, para el trabajo social, compartir los presupuestos teóricos y metodológicos de otras disciplinas como las ciencias sociales y beneficiarse con sus investigaciones y propuestas teóricas enriqueciendo su práctica.

Para efectos del presente escrito, y para concluir este apartado, baste exponer la visión netamente humana y contemporánea del trabajo social, definida por Flores y Martínez (2006):
La visión filosófica humana en esta idea contemporánea en que concebimos un trabajo social alternativo, se separa de la perspectiva religiosa en sus distintas vertientes, que dieron sustento a las acciones que precedieron las épocas del Trabajo Social profesional: caridad, filantropía y de algún modo asistenciales. La visión contemporánea que asumimos requiere de un proyecto de nación democrático que incluya el ejercicio de los derechos sociales, civiles y humanos, de los cuales debe ser vigilante la función profesional, además de considerar la subjetividad, ámbito en el que están los proyectos de autonomía y autodeterminación del sujeto-actor social. (p. 257).

En esta idea sobre el trabajo social se retoma lo que considero el espíritu de la disciplina y la evolución epistémica que ha tenido a largo de su historia. No basta únicamente con separar la perspectiva religiosa que precedió al trabajo social profesional, fundamentada en la caridad y la filantropía de algún modo asistenciales, sino que se requiere de un propósito firme por constituir una sociedad democrática que incluya el ejercicio de los derechos sociales, civiles y humanos de todos sus integrantes. 


\section{Historia Oral}

Por su parte, la historia oral utiliza la entrevista registrada en audio o video como constituyente fontal para acercarnos al pasado reciente. El trabajo social y la sociología pueden utilizar la entrevista para la interpretación del presente, de tal suerte que los testimonios orales y sus transcripciones alcanzan la categoría de documentos válidos para el estudio del pasado reciente, pero también, de la sociedad actual.

La llamada historia oral se muestra, por un lado, como una propuesta de método adecuado para estudiar la historia de los grupos subalternos y como uno de los métodos más pertinentes para recoger y conocer la "voz propia" de los sujetos históricos y los hechos sociales, o las experiencias humanas que han interesado a los investigadores (Aceves, 1996). Por otro lado, siguiendo a Plummer (1989), el objetivo de la historia oral es obtener información sobre el pasado, pero también producir conocimientos históricos y científicos, y no simplemente establecer una relación sistemática de la vida y experiencia de los "otros".

Es incuestionable que la historia oral no encaja en los presupuestos clásicos, en los cuales la evidencia puede ser manipulada pero nunca fabricada, ya que en la historia oral la evidencia se hace en el sentido que es el resultado del discurso subjetivo del entrevistado, guiado

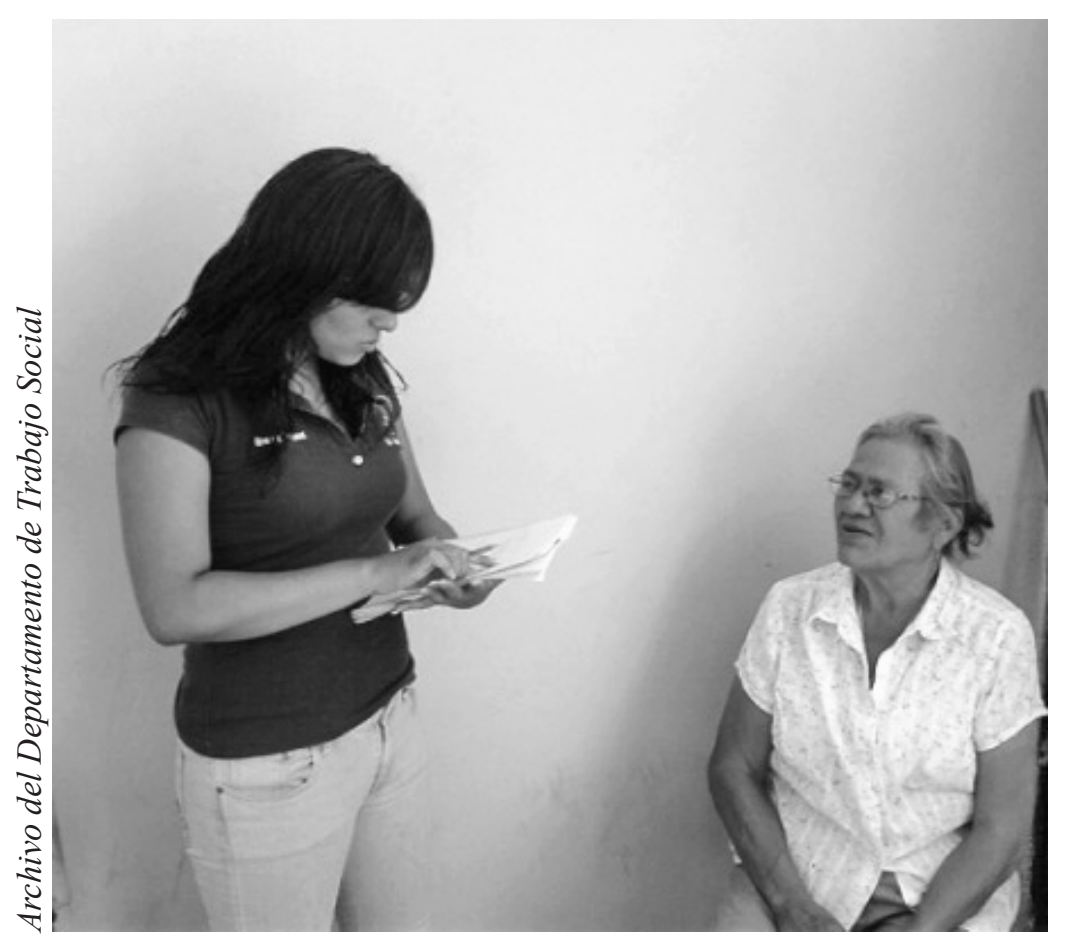

a su vez por las cuestiones planteadas subjetivamente por el entrevistador (Gil, 1998). Esta subjetividad, lejos de menoscabar la validez del discurso elaborado, debemos considerarla como el umbral, es decir, el sitio de arranque o punto de partida para la interpretación/ comprensión de la sociedad en la que vivimos, así como de sucesos pasados que pretendemos reconstruir.

La historia oral puede darnos una forma eficaz de aproximarnos a la cotidianidad de las personas, tan escasamente registrada con formas habituales de recogida de datos. Asimismo nos muestra, de "viva voz", las configuraciones y puntos de vista que tienen los protagonistas de su experiencia individual y colectiva; en todo caso nos revela el sentir, digamos pálpito a pálpito, de la vida cotidiana.

La historia oral se vitalizó cuando estudiosos e investigadores la utilizaron para darle voz a los "sin voz"; cuando el sentir y hacer de los estratos más desprotegidos y vilipendiados de la sociedad podían levantar sus palabras y registrarlas en esta nueva forma de hacer historia: alejadas del frío e impersonal documento de archivo, las palabras imprimen a la historia un aliento vital. (Thompson, 1988).

Sin embargo, la historia oral no es necesariamente un instrumento de cambio pero puede ser, y en muchos casos los es, un medio de transformar, tanto el contenido como el propósito de la historia. A través de la historia oral se puede dar a la comunidad la seguridad de escribir su propia historia.

Asimismo, y como asunto importante para este escrito, enfatizo que la historia oral se muestra, por su carácter creativo y cooperativo, adaptable a otras disciplinas de las ciencias sociales en la elaboración y puestas en escena de proyectos multidisciplinarios conjuntos.

\section{Proyectos}

Thompson, pionero de la historia oral, en su célebre trabajo La voz del pasado: la historia oral (1988), dedica un capítulo entero para mostrar el carácter creativo y cooperativo de la historia oral. Los proyectos de historia oral resultan posibles de realizar en un sinfín de contextos diferentes, tanto individualmente como en grupo. Ya sea en escuelas primarias o en universidades, en hospitales o centros penitenciarios, en clubes de 
amigos o en centros de atención y cuidados para adultos mayores, etcétera, se puede recoger testimonio oral.

En tales proyectos, apunta la tesis de este artículo, profesionales de otras disciplinas pueden adecuar los fines y objetivos de su trabajo y "enriquecer" los resultados en la solución de problemas planteados desde su propia disciplina, es decir, en el caso del trabajo social, la intervención en asuntos puntuales, como elevar la calidad de vida de los llamados adultos mayores o de la tercera edad albergados o recluidos en residencias geriátricas, los llanamente denominados asilos de ancianos. En estos lugares seguramente se alcanzarían resultados boyantes a partir de las herramientas metodológicas propuestas por la historia oral.

Comentamos al principio de este escrito que el instrumento por antonomasia de la historia oral es la entrevista. La entrevista - siguiendo a Thompson (Ibíd.) - implica que el hablante haga una rememoración de su vida o parte de ella. El psicoanálisis, por ejemplo, ensalza la rememoración a un modelo terapéutico. El recordar la propia vida es crucial para el sentido de sí mismo, de tal suerte que ejercitar la memoria puede fortalecer o recobrar la confianza de los entrevistados; además, la rememoración permite reevaluar los conflictos pasados y restablecer la propia identidad y un medio de ayudar, en el supuesto caso esgrimido líneas arriba, a los ancianos a valerse por sí mismos. A través de la entrevista las personas de la tercera edad "pueden reflexionar sobre sus vidas con el ánimo de resolver, reorganizar y reintegrar aquello que les cause preocupaciones o problemas" (p. 182). Los llamados adultos mayores, aquellas personas con varios costales de años a cuestas, al igual que todos, necesitamos la oportunidad de expresar sentimientos.

En un proyecto de este tipo, realizado por trabajadores sociales o estudiantes de la profesión en prácticas o cumpliendo su servicio social, por medio de la entrevista, individual o grupal, podrán obtener productos directos para objetivos concretos; es decir, a partir del material grabado y transcrito, socializado en sesiones realizadas periódicamente. Podrían crear teatro a partir de las memorias de las personas mayores y luego representarlas en una audiencia compuesta por los propios personajes que inspiraron los diálogos. Podrían editar revistas y folletos con los resultados de las entrevistas con temas diversos, surgidos del propio interés de los entrevistados. Por ejemplo, trabajos comunes de antaño, cómo era la ciudad de origen, el comercio, la escuela, la familia, la niñez, el campo, la música, los bailes, el noviazgo, el matrimonio, la religión, la participación política, etcétera.

Los productos de las entrevistas podrán ir acompañados de fotografías y otras ilustraciones, la comunicación verbal entre los ancianos participantes se vería favorecida ya que se aportarían temas de conversación común y, "una vez iniciada la comunicación, las personas se redescubren como seres humanos", es decir, se enriquecería la calidad de vida con betas de momentos felices y plenos: "escuchar seriamente lo que los ancianos tengan que decir, es la manera en que un persona vieja, miserable, difícil y quejumbrosa puede pasar a ser plenamente una persona." (p. 186). Nos recuerda Thompson en la obra antes citada.

Estoy convencido que los proyectos de historia oral en manos de trabajadores sociales multiplicarían los resultados benignos de la intervención. Los productos acabados y obtenidos de la puesta en escena de proyectos de este tipo podrían sistematizarse, analizarse, interpretarse y dar lugar a tesis y demás trabajos de investigación, o dicho con palabras de Bertaux, podrían ser sometidos a la exploración, el análisis y la expresión (síntesis) en forma oral y escrita con la finalidad de difundirlos.

\section{Colofón}

A manera de conclusión. Insistir en algunas ideas expuestas a lo largo de estas breves reflexiones: el trabajo social, como las demás disciplinas de las ciencias sociales, se esfuerza por la constitución de su estatuto de, digamos cientificidad. A lo largo de su historia el trabajo social ha logrado la construcción, más o menos acabada, de su sistematización y se ha beneficiado al compartir los presupuestos teóricos y metodológicos de las otras disciplinas de las ciencias sociales, no obstante, siempre ha sido el apego a los principios de los derechos humanos y la justicia social a favor de los más necesitados un objetivo sustantivo.

En la actualidad, estudiosos de las ciencias sociales han estado redescubriendo el valor de la rememoración en la entrevista en profundidad, pero bajo diferentes búsquedas. También, ante el desencanto del grosero empirismo masivo de la escuela cuantitativa, las entrevistas dirigidas por el método biográfico o la historia oral, utilizadas desde el trabajo social, parecen 


\section{就 Sorvia}

convidar información válida para utilizarse en un sinnúmero de proyectos cuya finalidad sea resolver problemas concretos que tengan que ver con el aumento en la calidad de vida de distintos grupos sociales vulnerables, por citar un ejemplo.

Para finalizar estas breves reflexiones, insistir en lo que considero también importante, los productos de los proyectos de historia oral, nos otorgarían abundancia de información capaz de ser analizada e interpretada desde la propia disciplina y con el fin de realizar proyectos de investigación.

\section{Bibliografía}

Aceves Lozano, J.E. (1996). Historia oral e historias de vida (Teoría, métodos y técnicas. Una bibliografía comentada). México: Colección Miguel Othón de Mendizábal, Ediciones de la Casa Chata, Ciesas, Hidalgo y Matamoros.

Bertaux, D. (1989). "Los relatos de vida en el análisis", Historia y fuente oral, No. 1. Barcelona: Publicación Universidad de Barcelona (2da Época), pp. 87-96.
Del Valle, A. H. y Ramella, M. S. (s/f). De Trabajo Social y Ciencias Sociales: de la reconceptualización al método crítico. Un viaje de vida. Recuperado en: http://revinut.udea.edu.co/index.php/revistraso/ article/viewFile/5274/4633..

Flores, C. y Martínez, G. (2006) "Hacia una concepción del Trabajo Social contemporáneo en México: su condición profesional". Katálysis, Vol. 9, No. 2, pp. 249-259.

Gil, F. (1998). "Posestructuralismo e historia oral". Historia, antropología y fuentes orales, No. 19. Barcelona: Publicación Universidad de Barcelona (2da Época), pp. 117-126.

Plummer, K. (1989). Los documentos personales (Introducción a los problemas y la bibliografía del método humanista. España: Siglo XXI Editores, S.A.

Thompson, P. (1988). La voz del pasado: la historia oral. Valencia: Alfons El Magnànim.

(s/f). Historias de vida y análisis del cambio social. Módulo Virtual: Memorias de la Violencia. Recuperado en: http://www.cholonautas.edu.pe/ memoria/thompson4.pdf.

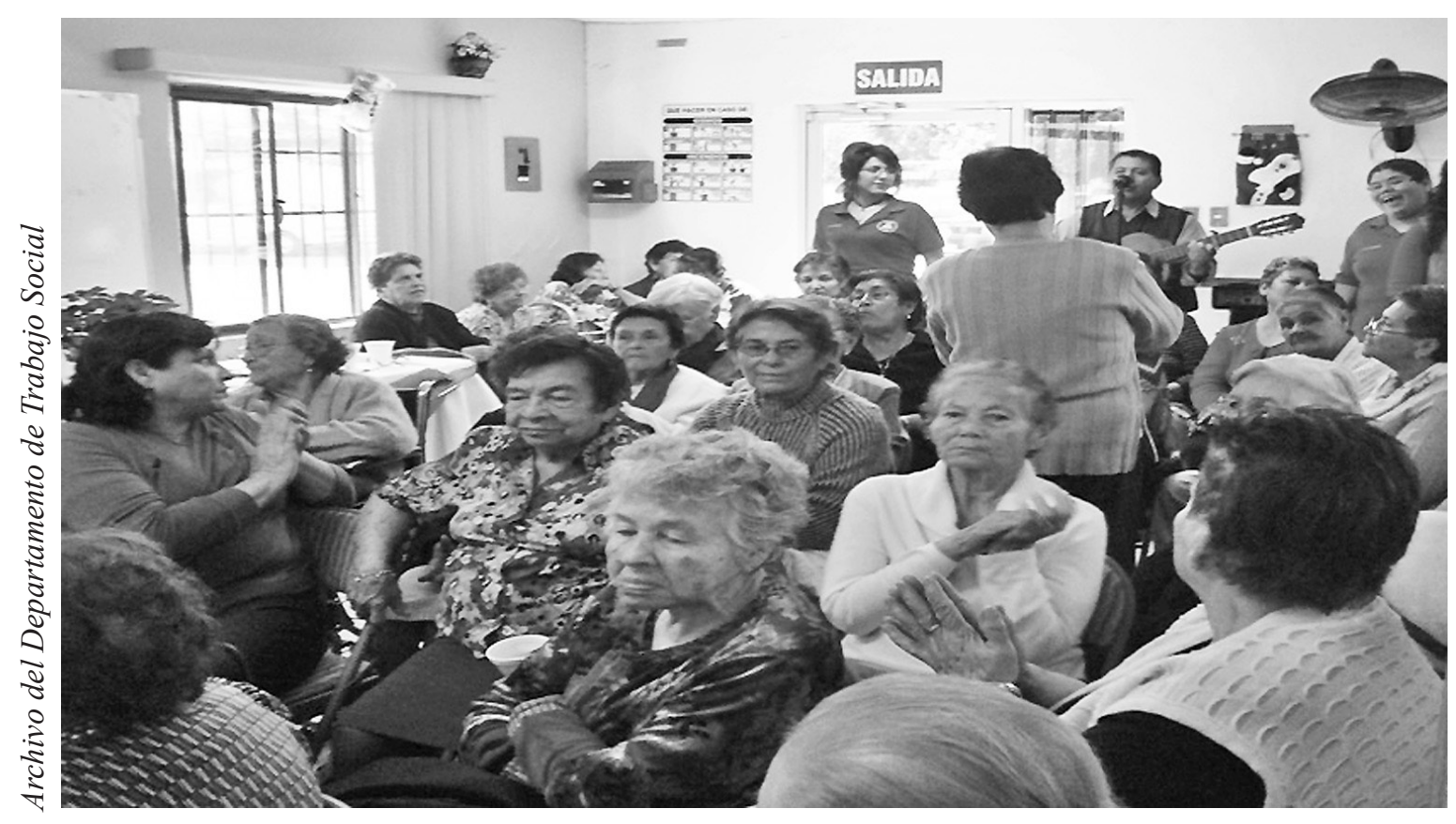

